\title{
FIELDS OF DIMENSION ONE ALGEBRAIC OVER A GLOBAL OR LOCAL FIELD NEED NOT BE OF TYPE $C_{1}$
}

\author{
IVAN D. CHIPCHAKOV
}

\begin{abstract}
Let $(K, v)$ be a Henselian discrete valued field with a quasifinite residue field. This paper proves the existence of an algebraic extension $E / K$ satisfying the following: (i) $E$ has $\operatorname{dimension} \operatorname{dim}(E) \leq 1$, i.e. the Brauer group $\operatorname{Br}\left(E^{\prime}\right)$ is trivial, for every algebraic extension $E^{\prime} / E$; (ii) finite extensions of $E$ are not $C_{1}$-fields. This, applied to the maximal algebraic extension $K$ of the field $\mathbb{Q}$ of rational numbers in the field $\mathbb{Q}_{p}$ of $p$-adic numbers, for a given prime $p$, proves the existence of an algebraic extension $E_{p} / \mathbb{Q}$, such that $\operatorname{dim}\left(E_{p}\right) \leq 1, E_{p}$ is not a $C_{1}$-field, and $E_{p}$ has a Henselian valuation of residual characteristic $p$.
\end{abstract}

\section{Introduction}

A field $F$ is said to be of dimension $\leq 1$, if the Brauer groups $\operatorname{Br}\left(F^{\prime}\right)$ are trivial, for all algebraic field extensions $F^{\prime} / F$. It is known (cf. 29], Ch. II, 3.1) that $\operatorname{dim}(F) \leq 1$ if and only if $\operatorname{Br}\left(F^{\prime}\right)=\{0\}$, where $F^{\prime}$ runs across the set $\mathrm{Fe}(F)$ of finite extensions of $F$ in its separable closure $F_{\text {sep. }}$. When $F$ is perfect, we have $\operatorname{dim}(F) \leq 1$ if and only if the absolute Galois group $\mathcal{G}_{F}:=\mathcal{G}\left(F_{\text {sep }} / F\right)$ has cohomological dimension $\operatorname{cd}\left(\mathcal{G}_{F}\right) \leq 1$ as a profinite group. For example, we have $\operatorname{dim}(F) \leq 1$ in case $F$ is a quasifinite field, i.e. a perfect field which admits, for each $n \in \mathbb{N}$, a unique extension in $F_{\text {sep }}$ of degree $n$. It is well-known that then $\mathcal{G}_{F}$ is isomorphic to $\mathcal{G}_{\mathbb{F}}$, for any finite field $\mathbb{F}$, i.e. it is a profinite completion of the additive group $\mathbb{Z}$ of integers. One has $\operatorname{cd}\left(\mathcal{G}_{F}\right) \leq 1$, since $\mathcal{G}_{F}$ is isomorphic to the topological group product $\prod_{p \in \mathbb{P}} \mathbb{Z}_{p}$, where $\mathbb{P}$ is the set of prime numbers and $\mathbb{Z}_{p}$ is the additive group of $p$-adic integers, for each $p$ (see [17, Examples 4.1.2).

We say that $F$ is of type $C_{r}$ (or a $C_{r}$-field), for some $r \in \mathbb{N}$, if every $F$ form (a homogeneous nonzero polynomial with coefficients in $F$ ) $f$ of degree $\operatorname{deg}(f)$ in more than $\operatorname{deg}(f)^{r}$ variables has a nontrivial zero over $F$. For $F$ to be a $C_{r}$-field, it is necessary that $\operatorname{char}(F)=q$, and in case $q>0$, the degree $\left[F: F^{q}\right]$ of $F$ as an extension of its subfield $F^{q}=\left\{\beta^{q}: \beta \in F\right\}$ be at most equal to $q^{r}$. The class of $C_{r}$-fields is closed under the formation of algebraic extensions, and it contains the extensions of transcendency degree $r$ over any algebraically closed field (cf. [22]). It is known that $C_{1}$-fields have dimension $\leq 1$ but the converse is not necessarily true in nonzero characteristic; one can take as a counter-example any field $\Phi$ with $\operatorname{char}(\Phi)=q>0,\left[\Phi: \Phi^{q}\right]>q$ and $\Phi_{\text {sep }}=\Phi$ (see [29], Ch. II, 3.1 and 3.2). The restriction on the characteristic has been lifted by Ax [3], by providing an example of a quasifinite field $F$ (so

Key words and phrases. Field of dimension $\leq 1$, field of type $C_{1}$, form, Henselian valuation

2020 MSC Classification: 11E76 11R34 (primary), 12F10, 12J10, 11S15 (secondary). 
having $\operatorname{dim}(F) \leq 1$ ), such that $\operatorname{char}(F)=0$ and $F$ is not a $C_{r}$-field, for any $r \in \mathbb{N}$. This example is in sharp contrast to the Chevalley-Warning theorem (see, e.g., [17, Theorem 6.2.6), which establishes the $C_{1}$ type of finite fields.

As noted by Serre in [29], Ch. II, 3.3, it is not known whether an algebraic extension $E$ of the field $\mathbb{Q}$ of rational numbers is of type $C_{1}$, provided that $\operatorname{dim}(E) \leq 1$; he has added that this is not likely to hold in general.

The present paper answers the question arising from Serre's remark, in the direction pointed there. The answer is unchanged when $\mathbb{Q}$ is replaced by any global or local field. This is obtained by methods of valuation theory, using the fact that nontrivial Krull valuations of global fields are discrete with finite residue fields (see [12], Examples 4.1.2, 4.1.3 and Corollary 14.2.2). The considered question remains open for Galois extensions $E$ of $\mathbb{Q}$ with $\operatorname{dim}(E) \leq 1$.

\section{Statements of the main results}

The main results of this paper are presented as two theorems. The former theorem is a special case of the latter one and can be stated as follows:

Theorem 2.1. For each prime number $p$, there exists an algebraic extension $E_{p}$ of the field $\mathbb{Q}$ of rational numbers, such that $\operatorname{dim}\left(E_{p}\right) \leq 1$, the finite extensions of $E_{p}$ are not $C_{1}$-fields, and $E_{p}$ is endowed with a Henselian valuation $v$ whose residue field $\widehat{E}_{p}$ is of characteristic $p$.

As usual, by a Henselian valuation of a field $K$, we mean a nontrivial Krull valuation $v$ that extends uniquely, up-to equivalence, to a valuation $v_{L}$ on each algebraic extension $L$ of $K$. When $v$ is Henselian, $(K, v)$ is called a Henselian field. Our next result, stated below, shows that the class of separable (algebraic) extensions of Henselian discrete valued 1 fields with finite residue fields contains fields of dimension $\leq 1$, which are not of type $C_{1}$.

Theorem 2.2. Let $(K, v)$ be an HDV-field with a quasifinite residue field. Then there exists an extension $E$ of $K$ in $K_{\mathrm{sep}}$, such that $\operatorname{dim}(E) \leq 1$ and finite extensions of $E$ are not $C_{1}$-fields. Moreover, $E$ can be chosen so that there is a sequence $f_{n}, n \in \mathbb{N}$, of E-forms subject to the following restrictions:

(a) $f_{n}$ does not possess a nontrivial zero over $E$, for any index $n$;

(b) The degrees $\operatorname{deg}\left(f_{n}\right):=p_{n}, n \in \mathbb{N}$, form a strictly increasing sequence of prime numbers, and for each $n, f_{n}$ depends essentially on exactly $p_{n} k_{n}$ variables, for some $k_{n} \in \mathbb{N}$ with $2 \leq k_{n} \leq\left(p_{n}-1\right) / 2$.

Theorem 2.2 is proved in Section 4. Here we show that Theorem 2.2 implies Theorem 2.1. Denote by $\mathbb{P}$ the set of prime numbers, and for each $p \in \mathbb{P}$, let $K_{p}$ be the maximal separable extension of $\mathbb{Q}$ in the field $\mathbb{Q}_{p}$ of $p$ adic numbers. It is known (cf. [12], Theorem 15.3.5) that the valuation, say $\omega_{p}$, induced on $K_{p}$ by the standard valuation of $\mathbb{Q}_{p}$ is Henselian and discrete,

\footnotetext{
${ }^{1}$ In what follows, we write briefly "HDV" instead of "Henselian discrete valued".
} 
and the residue field $\widehat{K}_{p}$ of $\left(K_{p}, \omega_{p}\right)$ is a field with $p$ elements. Hence, by Theorem 2.2, it has an algebraic extension $E_{p}$ with the properties required by Theorem 2.1 . The question of whether an algebraic extension $E$ of $\mathbb{Q}$ with $\operatorname{dim}(E) \leq 1$ is a $C_{\nu}$-field, for some integer $\nu \geq 2$, remains open. Note in this connection that examples given by Arkhipov and Karatsuba [2] (see also [6], Ch. I, Sect. 6.5, and further references there) show that $\mathbb{Q}_{p}, p \in \mathbb{P}$, are not $C_{\nu}$-fields, for any $\nu \in \mathbb{N}$.

Similarly, if $\mathbb{F}_{p}(X)$ is the rational function field in a variable $X$ over the field $\mathbb{F}_{p}$ with $p$ elements, $K_{p}$ is the maximal separable extension of $\mathbb{F}_{p}(X)$ in the formal Laurent power series field $\mathbb{F}_{p}((X))$, and $\omega_{p}$ is the valuation of $K_{p}$ induced by the natural discrete valuation of $\mathbb{F}_{p}((X))$, then $\left(K_{p}, \omega_{p}\right)$ is an HDV-field. Therefore, any extension $E_{p}$ of $K_{p}$ in $K_{p \text {,sep }}$ with the properties claimed by Theorem 2.2 is an algebraic extension of $\mathbb{F}_{p}(X)$, such that $\operatorname{dim}\left(E_{p}\right) \leq 1$ and finite extensions of $E_{p}$ are not $C_{1}$-fields $\left(E_{p}\right.$ is a $C_{2^{-}}$ field, by [22], Theorem 8). Note here that $\left[E_{p}: E_{p}^{p}\right]=p$ (see (3.2) (a) and $(3.5)\left(\right.$ b)), i.e. $E_{p}$ satisfies the necessary condition for having type $C_{1}$, stated in Section 1 (and proved in [29], Ch. II, 3.2). These facts (see also (3.5) (a) and the proof of (3.5)) show that $C_{1}$-fields, global fields and local fields are almost perfect, in the following sense:

A field $E$ is called almost perfect if one of the following two equivalent conditions holds: (i) every finite extension of $E$ has a primitive element; (ii) $\operatorname{char}(E)=q$, and in case $q>0,\left[E: E^{q}\right]$ equals 1 or $q$ (the equivalence of conditions (i) and (ii) is implied by [24], Ch. V, Theorem 4.6 and Corollary 6.10).

Theorem 2.2 and the method of proving Theorem 2.1 enable one to answer the main question considered in this paper, for Henselian fields that are algebraic extensions of an arbitrary global field $K$ (see Corollary 4.2). At the same time, the discussed question remains widely open over other interesting classes of algebraic extensions of $K$, e.g., the class $\mathcal{V}(K)$ of algebraic extensions $E / K$, such that $\mathcal{G}_{E}$ is a torsion-free group and Henselizations of $E$ in $E_{\text {sep }}$ with respect to any nontrivial valuation coincide with $E_{\text {sep. }}$. By the Artin-Schreier theory (cf. 24], Ch. XI, Theorem 2.2), the condition on $\mathcal{G}_{E}$ restates the one that $E$ is a nonreal field, i.e. -1 is presentable over $E$ as a finite sum of squares; its violation yields $\operatorname{Br}(E) \neq\{0\}$ (the equivalence class in $\operatorname{Br}(E)$ of the $E$-algebra of Hamiltonian quaternions is an element of order 2). When $E$ is nonreal, the description of $\operatorname{Br}(K)$ by class field theory (cf. [31, Ch. XIII, Sects. 3, 6) enables one to obtain that $\operatorname{dim}(E) \leq 1$ by the method of proving Proposition 9 of [29], Ch. II. Also, by the FreyPrestel theorem (see [14], Theorem 2, and [15], Corollary 11.5.5), PAC2 fields are contained in the class $\mathcal{V}$ of nonreal fields whose Henselizations with respect to any nontrivial valuation are separably closed. The main result of [16] shows the existence of non-PAC fields in $\mathcal{V}$ and raises interest in the following open problem (see Remark 4.4 and [15], Problem 11.5.9 (b)):

Problem 2.3. For a global field $K$, find whether all $\Phi \in \mathcal{V}(K)$ are $P A C$ fields.

\footnotetext{
${ }^{2}$ As usual, PAC is an abbreviation for "pseudo algebraically closed".
} 
The basic notation, terminology and conventions kept in this paper are standard and virtually the same as in [30, [24] and [7]. Throughout, Brauer and value groups are written additively, Galois groups are viewed as profinite with respect to the Krull topology, and by a profinite group homomorphism, we mean a continuous one. For any field $E, E^{*}$ is its multiplicative group, $E^{* n}=\left\{a^{n}: a \in E^{*}\right\}$, for each $n \in \mathbb{N}$, and $\operatorname{Br}(E)_{p}, p \in \mathbb{P}$, are the $p$ components of $\operatorname{Br}(E)$. As usual, for any $p \in \mathbb{P}, E(p)$ denotes the maximal $p$-extension of $E$ (in $E_{\mathrm{sep}}$ ), that is, the compositum of those finite Galois extensions of $E$ in $E_{\mathrm{sep}}$, whose Galois groups are $p$-groups. Given a field extension $E^{\prime} / E$, we write $\mathrm{I}\left(E^{\prime} / E\right)$ for the set of intermediate fields of $E^{\prime} / E$, $\pi_{E \rightarrow E^{\prime}}$ for the scalar extension map $\operatorname{Br}(E) \rightarrow \operatorname{Br}\left(E^{\prime}\right)$, and $\operatorname{Br}\left(E^{\prime} / E\right)$ for the relative Brauer group of $E^{\prime} / E$ (the kernel of $\pi_{E \rightarrow E^{\prime}}$ ). When $E^{\prime} / E$ is a finite extension, $N_{E^{\prime} / E}$ denotes the norm map $E^{\prime} \rightarrow E$, and $N\left(E^{\prime} / E\right)$ stands for the norm group of $E^{\prime} / E$ (the image of $E^{\prime *}$ under $N_{E^{\prime} / E}$ ). Moreover, if $E^{\prime} / E$ is a Galois extension, then its Galois group is denoted by $\mathcal{G}\left(E^{\prime} / E\right)$; we say that $E^{\prime} / E$ is a cyclic extension if $\mathcal{G}\left(E^{\prime} / E\right)$ is a cyclic group. By a

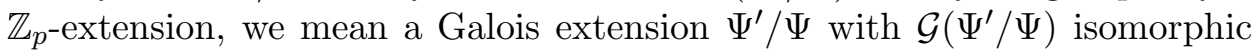
to $\mathbb{Z}_{p}$. The value group of any discrete valued field is assumed to be an ordered subgroup of the additive group of the field $\mathbb{Q}$; this is done without loss of generality, in view of [12], Theorem 15.3.5, and the fact that $\mathbb{Q}$ is a divisible hull of any of its infinite subgroups (see page 5 ).

Here is an overview of this paper: Section 3 includes valuation-theoretic preliminaries used in the sequel as well as characterizations of fields of dimension $\leq 1$ among algebraic extensions of local fields. As noted above, Theorem 2.2 is proved in Section 4. This is done by modifying the proof of the Theorem of [3], given in [4]; specifically, the forms violating the $C_{1}$ condition are defined by essentially the same pattern in both proofs.

\section{Preliminaries and characterizations of algebraic extensions $E$ of local fields with $\operatorname{Br}(E)_{p}=\{0\}$, for a given prime $p$}

For any field $K$ with a (nontrivial) Krull valuation $v, O_{v}(K)=\{a \in$ $K: v(a) \geq 0\}$ denotes the valuation ring of $(K, v), M_{v}(K)=\{\mu \in K: v(\mu)>$ $0\}$ the maximal ideal of $O_{v}(K), O_{v}(K)^{*}=\{u \in K: v(u)=0\}$ the multiplicative group of $O_{v}(K), v(K)$ the value group and $\widehat{K}=O_{v}(K) / M_{v}(K)$ the residue field of $(K, v)$, respectively; $\overline{v(K)}$ is a divisible hull of $v(K)$. The condition that $v$ is Henselian has the following two equivalent forms (cf. [12, Sect. 18.1):

(3.1) (a) Given a polynomial $f(X) \in O_{v}(K)[X]$ and an element $a \in O_{v}(K)$, such that $2 v\left(f^{\prime}(a)\right)<v(f(a))$, where $f^{\prime}$ is the formal derivative of $f$, there is a zero $c \in O_{v}(K)$ of $f$ satisfying the equality $v(c-a)=v\left(f(a) / f^{\prime}(a)\right)$;

(b) For each normal extension $\Omega / K, v^{\prime}(\tau(\mu))=v^{\prime}(\mu)$ whenever $\mu \in \Omega, v^{\prime}$ is a valuation of $\Omega$ extending $v$, and $\tau$ is a $K$-automorphism of $\Omega$.

Next we recall some facts concerning the case where $(K, v)$ is a real-valued field, i.e. $v(K)$ is embeddable as an ordered subgroup in the additive group $\mathbb{R}$ of real numbers. Fix a completion $K_{v}$ of $K$ with respect to the topology 
induced by $v$, and denote by $\bar{v}$ the valuation of $K_{v}$ continuously extending $v$. Then:

(3.2) (a) $(K, v)$ is Henselian if and only if $K$ has no proper separable (algebraic) extension in $K_{v}$ (cf. [12], Corollary 18.3.3);

(b) The topology of $K_{v}$ as a completion of $K$ is the same as the one induced by $\bar{v}$; also, $\bar{v}\left(K_{v}\right)=v(K), \widehat{K}$ equals the residue field of $\left(K_{v}, \bar{v}\right)$, and $\left(K_{v}, \bar{v}\right)$ is a Henselian field (cf. [12, Theorems 9.3.2 and 18.3.1).

When $v$ is Henselian (but not necessarily real-valued), so is $v_{L}$, for any algebraic field extension $L / K$. In this case, we denote by $\widehat{L}$ the residue field of $\left(L, v_{L}\right)$, and put $O_{v}(L)=O_{v_{L}}(L), M_{v}(L)=M_{v_{L}}(L), v(L)=v_{L}(L)$; also, we write $v$ instead of $v_{L}$ when there is no danger of ambiguity. Clearly, $\widehat{L} / \widehat{K}$ is an algebraic extension and $v(K)$ is an ordered subgroup of $v(L)$, such that $v(L) / v(K)$ is a torsion group; hence, one may assume without loss of generality that $v(L)$ is an ordered subgroup of $\overline{v(K)}$. By Ostrowski's theorem (cf. 12], Theorem 17.2.1), if $[L: K]$ is finite, then it is divisible by $[\widehat{L}: \widehat{K}] e(L / K)$, and in case $[L: K] \neq[\widehat{L}: \widehat{K}] e(L / K)$, the integer $[L: K][\widehat{L}: \widehat{K}]^{-1} e(L / K)^{-1}$ is a power of $\operatorname{char}(\widehat{K})($ so $\operatorname{char}(\widehat{K}) \mid[L: K])$; here $e(L / K)$ is the ramification index of $L / K$, i.e. the index of $v(K)$ in $v(L)$. Ostrowski's theorem implies the following:

(3.3) The quotient groups $v(K) / p v(K)$ and $v(L) / p v(L)$ are isomorphic, if $p \in \mathbb{P}$ and $[L: K]<\infty$. Moreover, if $\operatorname{char}(\widehat{K}) \nmid[L: K]$, then the natural embedding of $K$ into $L$ induces canonically an isomorphism $v(K) / p v(K) \cong$ $v(L) / p v(L)$.

The finite extension $L / K$ satisfies the equality $[L: K]=[\widehat{L}: \widehat{K}] e(L / K)$ in each of the following two situations:

(3.4) (a) $(K, v)$ is HDV and $L / K$ is separable (see [12], Sect. 17.4).

(b) $(K, v)$ is HDV and the field $K$ is almost perfect (cf. [24], Ch. XII, Proposition 6.1). When $\operatorname{char}(K)=q>0$, this implies $\left[K: K^{q}\right]=q$, since $K^{q}$ does not contain any $\alpha \in K^{*}$ with $v(\alpha) \notin q v(K)$.

We show that the conditions of (3.4) (b) hold in the following two cases: (3.5) (a) $(K, v)$ is a complete discrete valued field (i.e. $(K, v)=\left(K_{v}, \bar{v}\right)$ and $v$ is discrete) with $\widehat{K}$ perfect.

(b) $(K, v)$ is HDV and $K$ is an algebraic extension of a global field $K_{0}$ (see [12], Example 4.1.3).

Complete discrete valued fields are Henselian, by (3.2), so it suffices for the proof of (3.5) to show that $\left[K: K^{q}\right]=q$, provided that $\operatorname{char}(K)=q>0$. Under the hypotheses of (3.5) (a), $K$ is isomorphic to the formal Laurent power series field $\widehat{K}((X))$ in a variable $X$ over $\widehat{K}$ (see [12], Theorem 12.2.3). Since $\widehat{K}$ is perfect, whence, $\widehat{K}((X))^{q}=\widehat{K}\left(\left(X^{q}\right)\right)$, and $1, X, \ldots, X^{q-1}$ is a basis of $\widehat{K}((X))$ over $\widehat{K}\left(\left(X^{q}\right)\right)$, this yields $\left[K: K^{q}\right]=q$. Similarly, it turns out that if $K_{0}=\widehat{K}(X)$ and $\widehat{K}$ is the prime field $\mathbb{Z} / q \mathbb{Z}$, then $K_{0}^{q}=\widehat{K}\left(X^{q}\right)$ and $1, X, \ldots, X^{q-1}$ is a basis of $K_{0}$ over $K_{0}^{q}$. In view of [5], Lemma 2.12, this means that $\left[K_{0}: K_{0}^{q}\right]=q$, for any global field $K_{0}$ of characteristic $q$, and also proves (3.5) (b). 
Assume now that $(K, v)$ is a Henselian field and let $R$ be a finite extension of $K$. We say that $R / K$ is inertial, if $[R: K]=[\widehat{R}: \widehat{K}]$ and $\widehat{R}$ is separable over $\widehat{K} ; R / K$ is called totally ramified, if $e(R / K)=[R: K]$. Inertial extensions of $K$ are clearly separable; also, they have a number of useful properties, some of which are presented by the following lemma (for its proof, see [30], Theorem A.23):

Lemma 3.1. Let $(K, v)$ be a Henselian field and $K_{\mathrm{ur}}$ the compositum of inertial extensions of $K$ in $K_{\mathrm{sep}}$. Then:

(a) An inertial extension $R^{\prime} / K$ is Galois if and only if so is $\widehat{R}^{\prime} / \widehat{K}$. When this holds, $\mathcal{G}\left(R^{\prime} / K\right)$ and $\mathcal{G}\left(\widehat{R}^{\prime} / \widehat{K}\right)$ are canonically isomorphic. $\mathcal{G}_{\widehat{K}}$.

(b) $v\left(K_{\mathrm{ur}}\right)=v(K)$ and $K_{\mathrm{ur}} / K$ is a Galois extension with $\mathcal{G}\left(K_{\mathrm{ur}} / K\right) \cong$

(c) Finite extensions of $K$ in $K_{\mathrm{ur}}$ are inertial, and the natural mapping of $I\left(K_{\mathrm{ur}} / K\right)$ into $I\left(\widehat{K}_{\mathrm{sep}} / \widehat{K}\right)$, by the rule $L \rightarrow \widehat{L}$, is bijective.

The next two lemmas enable one to generalize a number of results on complete real-valued fields to the case of Henselian real-valued fields.

Lemma 3.2. Let $(K, v)$ be a real-valued field, $\left(K_{v}, \bar{v}\right)$ its completion, and $\left(K^{\prime}, v^{\prime}\right)$ an intermediate valued field of $\left(K_{v}, \bar{v}\right) /(K, v)$. Suppose that $\left(K^{\prime}, v^{\prime}\right)$ is Henselian, identify $K_{\mathrm{sep}}^{\prime}$ with its $K^{\prime}$-isomorphic copy in $K_{v \text {,sep }}$, and denote by $f$ the mapping $f: \mathrm{Fe}\left(K^{\prime}\right) \rightarrow \mathrm{Fe}\left(K_{v}\right)$, defined by the rule $\Lambda^{\prime} \rightarrow \Lambda^{\prime} K_{v}$. Then:

(a) $K_{\mathrm{sep}}^{\prime} \cap K_{v}=K^{\prime}$, and each $\Lambda \in \mathrm{Fe}\left(K_{v}\right)$ contains a primitive element $\lambda \in K_{\text {sep }}^{\prime}$ over $K_{v}$, such that $\left[K_{v}(\lambda): K_{v}\right]=\left[K^{\prime}(\lambda): K^{\prime}\right]$;

(b) $K_{\text {sep }}^{\prime} K_{v}=K_{v \text {,sep }}$ and $\mathcal{G}_{K^{\prime}} \cong \mathcal{G}_{K_{v}}$;

(c) The mapping $f$ is bijective and degree-preserving. Moreover, $f$ and the inverse mapping $f^{-1}: \mathrm{Fe}\left(K_{v}\right) \rightarrow \mathrm{Fe}\left(K^{\prime}\right)$, preserve the Galois property and the isomorphism class of the corresponding Galois groups.

Proof. The conditions on $(K, v)$ and the Henselian property of $\left(K^{\prime}, v^{\prime}\right)$ ensure that $K_{\text {sep }}^{\prime} \cap K_{v}=K^{\prime}$. The latter part of Lemma 3.2 (a) can be deduced from Krasner's lemma (see [23], Ch. II, Propositions 3, 4). The conclusions of Lemma 3.2 (c) follow from Lemma 3.2 (a) and Galois theory (cf. [24], Ch. VI, Theorem 1.12), and those of Lemma 3.2 (b) follow from Lemma 3.2 (a), (c) and the definition of the Krull topology on $\mathcal{G}_{K^{\prime}}$ and $\mathcal{G}_{K_{v}}$.

Lemma 3.3. Let $(K, v)$ be a Henselian real-valued field, $\left(K_{v}, \bar{v}\right)$ its completion, and $R$ an extension of $K$ in $K_{\text {sep }}$. Identify $K_{\text {sep }}$ with its $K$-isomorphic copy in $R_{v \text {,sep }}$, where $R_{v}=R_{v_{R}}$, and $K_{v}$ with the topological closure of $K$ in $R_{v}$, and put $R^{\prime}=K_{v} R$. Then $\left(R^{\prime}, \bar{v}_{R^{\prime}}\right)$ is an intermediate valued field of $\left(R_{v}, \overline{v_{R}}\right) /\left(R, v_{R}\right)$.

Proof. It follows from Lemma 3.2 (a), (c) and the Henselian property of $(K, v)$ that the mapping $\mathrm{Fe}(K) \rightarrow \mathrm{Fe}\left(K_{v}\right)$, by the rule $\Lambda \rightarrow K_{v} \Lambda$, is bijective and degree-preserving. This implies that, for each $\Lambda \in \mathrm{Fe}(K)$, the 
restriction of the norm map $N_{K_{v} \Lambda / K_{v}}$ on $\Lambda$ equals $N_{\Lambda / K}$, which shows that $\left(K_{v} \Lambda, \bar{v}_{K_{v} \Lambda}\right) /\left(\Lambda, v_{\Lambda}\right)$ is a valued field extension (see, e.g., [30], Lemma 1.6). At the same time, observing that $\left(K_{v}, \bar{v}\right)$ is Henselian and $K_{v} \Lambda$ is a completion of $\Lambda$ with respect to the topology of $v_{\Lambda}$ (see [24], Ch. XII, Proposition 3.1), one obtains that if $\Lambda$ is a finite extension of $K$ in $R$, then $\overline{v_{R}}$ extends $\bar{v}_{K_{v} \Lambda}=\bar{v}_{\Lambda}$ upon $R_{v}$. As $R^{\prime}$ equals the union $\cup K_{v} \Lambda$, when $\Lambda$ runs across the set of finite extensions of $K$ in $R$, these facts prove Lemma 3.3 .

Next we present characterizations of those fields of dimension $\leq 1$, which lie in the class of algebraic extensions of any HDV-field $(K, v)$ with $\widehat{K}$ quasifinite. They are stated as two lemmas. For a proof of the former one in the case where $(K, v)$ is a local field with $\operatorname{char}(K)=0$, see [29], Ch. II, 5.6, Lemma 3.

Lemma 3.4. Let $(K, v)$ be an $\mathrm{HDV}$-field with $\widehat{K}$ quasifinite, and let $R / K$ be an algebraic field extension. Fix some $p \in \mathbb{P}$, and in case $p=\operatorname{char}(K)$, suppose that $R / K$ is separable or $K$ is an almost perfect field. Then $\operatorname{Br}(R)_{p}=$ $\{0\}$ if and only if one of the following three equivalent conditions is fulfilled:

(a) $\operatorname{Br}\left(R^{\prime}\right)_{p}=\{0\}$, for every algebraic extension $R^{\prime} / R$; this holds if and only if $\operatorname{Br}\left(R^{\prime}\right)_{p}=\{0\}$, when $R^{\prime}$ runs across the set $\mathrm{Fe}(R)$;

(b) For any pair $R_{1}^{\prime} \in \mathrm{Fe}(R), R^{\prime} \in I\left(R_{1}^{\prime} / R\right)$, $p$ does not divide the period of the quotient group of $R^{\prime *}$ by the norm group $N\left(R_{1}^{\prime} / R^{\prime}\right)$ of the extension $R_{1}^{\prime} / R^{\prime}$

(c) There exists is a sequence $R_{n}, n \in \mathbb{N}$, of finite extensions of $K$ in $R$, such that $p^{n}$ divides the degree $\left[R_{n}: K\right]$, for each index $n$.

Proof. Our starting point is the fact that $K$ is a quasilocal field, in the sense of [7, which implies the following:

(3.6) For any pair $\Lambda, \Lambda^{\prime}$ of finite extensions of $K$, such that $\Lambda \in I\left(\Lambda^{\prime} / K\right)$ and $\Lambda^{\prime}$ is separable over $\Lambda$, the group $\operatorname{Br}\left(\Lambda^{\prime} / \Lambda\right)$ consists of all elements of $\operatorname{Br}(\Lambda)$ of orders dividing $\left[\Lambda^{\prime}: \Lambda\right]$ (see [28], Ch. XIII, Sect. 3; [7, Corollary 8.5); the assertion holds without the assumption that $\Lambda^{\prime} / \Lambda$ is separable, provided that $K$ is an almost perfect field (cf. [7], Corollary 8.6).

Note also that $\operatorname{Br}(R)_{p}$ equals the union of the images of $\operatorname{Br}(\Lambda)_{p}$ under the scalar extension maps $\pi_{\Lambda \rightarrow R}$, when $\Lambda$ runs across the set $I_{0}(R / K)$ of finite extensions of $K$ in $R$ (cf., e.g., [7, (1.3)). This indicates that $\operatorname{Br}(R)_{p}=\{0\}$ if and only if $\operatorname{Br}(\Lambda)_{p} \subseteq \operatorname{Br}(R / \Lambda)$, for any $\Lambda \in I_{0}(R / K)$. Observe now that if $R_{n}, n \in \mathbb{N}$, are fields satisfying condition (c) of Lemma 3.4 with respect to $R / K$, then for each finite extension $\Lambda$ of $K$ in $R$, the sequence $\Lambda R_{n}, n \in \mathbb{N}$, has an infinite subsequence satisfying the same condition with respect to $R / \Lambda$. At the same time, the violation of condition (c) means that there is a finite extension $\Lambda_{1}$ of $K$ in $R$, such that $p$ does not divide the degree of any finite extension of $\Lambda_{1}$ in $R$. Therefore, by (3.6), $\pi_{\Lambda_{1} \rightarrow R}$ maps $\operatorname{Br}\left(\Lambda_{1}\right)_{p}$ injectively into $\operatorname{Br}(R)_{p}$. Since $\operatorname{Br}(K)$ is isomorphic to the quotient group $\mathbb{Q} / \mathbb{Z}$ (cf. [28], Ch. XIII, Sect. 3), whence, $\operatorname{Br}(L)_{p} \neq\{0\}$ for every finite extension $L / K$, these remarks enable one to deduce from (3.6) (under the hypothesis that $R_{n} / K, n \in \mathbb{N}$, are separable, or $K$ is almost perfect) the following: (i) conditions (c) and (a) of Lemma 3.4 are equivalent; (ii) 
condition (c) holds if and only if $\operatorname{Br}(R)_{p}=\{0\}$. For a proof of the equivalence of conditions (a) and (b), and of the former part of condition (a) to the latter one, we refer the reader to [29], Ch. II, 3.1, and [17], Theorem 6.1.8.

It remains to be seen that if $p \neq \operatorname{char}(K)$, then the assertions of the lemma hold, for any algebraic extension $R / K$. Let $R_{0}$ be the maximal separable extension of $K$ in $R$. It is well-known that if $R \neq R_{0}$, then $\operatorname{char}(K)=q>0$ and finite extensions of $R_{0}$ in $R$ are of $q$-primary degrees (cf. [24], Ch. V, Corollary 6.2). This enables one to prove that $\operatorname{Br}\left(R / R_{0}\right)$ is a subgroup of $\operatorname{Br}\left(R_{0}\right)_{q}$ (see [27], Sect. 13.4). Note finally that, by the Albert-Hochschild theorem (cf. [29], Ch. II, 2.2), $\pi_{R_{0} \rightarrow R}$ maps $\operatorname{Br}\left(R_{0}\right)_{p}$ surjectively upon $\operatorname{Br}(R)_{p}$. When $p \neq q$, these observations show that $\pi_{R_{0} \rightarrow R}$ induces an isomorphism $\operatorname{Br}\left(R_{0}\right)_{p} \cong \operatorname{Br}(R)_{p}$, so we have $\operatorname{Br}\left(R_{0}\right)_{p}=\{0\} \Leftrightarrow \operatorname{Br}(R)_{p}=$ $\{0\}$. The obtained equivalence completes the proof of Lemma 3.4, since its assertions apply to the extension $R_{0} / K$.

It is known that, for any field $R$, conditions (a) and (b) stated in Lemma 3.4 are equivalent, and when they hold, $\mathcal{G}_{R}$ is a profinite group of cohomological $p$-dimension $\operatorname{cd}_{p}\left(\mathcal{G}_{R}\right) \leq 1$; this implication is an equivalence in case $R$ is perfect or $p \neq \operatorname{char}(R)$ (cf. [29], Ch. II, 3.1, and [17], Theorem 6.1.8). Thus it follows that $\operatorname{dim}(R) \leq 1 \Leftrightarrow N\left(R_{1}^{\prime} / R^{\prime}\right)=R^{\prime *}$, for any pair $R_{1}^{\prime} \in \mathrm{Fe}(R)$, $R^{\prime} \in I\left(R_{1}^{\prime} / R\right)$ (cf. 29], Ch. II, 3.1). Clearly, $N\left(R_{1}^{\prime} / R^{\prime}\right)=R^{\prime *}$ if and only if the $R^{\prime}$-form $N_{R_{1}^{\prime} / R^{\prime}}\left(X_{1}, \ldots, X_{n}\right)-a X_{n+1}^{n}$ has a nontrivial zero over $R^{\prime}$, for each $a \in R^{\prime *}$, where $n=\left[R_{1}^{\prime}: R^{\prime}\right]$ and $N_{R_{1}^{\prime} / R^{\prime}}\left(X_{1}, \ldots, X_{n}\right)$ is the norm form of degree $n$ in algebraically independent variables $X_{1}, \ldots, X_{n}$ over $R^{\prime}$, associated with a fixed $R^{\prime}$-basis of $R_{1}^{\prime}$. This standardly proves the fact that $C_{1}$-fields have dimension $\leq 1$ (cf. [29], Ch. II, 3.2) and leads to the problem of whether the converse holds if one restricts to fields from special classes of sufficient research interest, such as the class of Henselian fields algebraic over a global field $K$, and the class $\mathcal{V}(K)$ defined in Section 2 (for other classes, see [20, Theorem B, and [26]).

Lemma 3.5. Let $(K, v)$ be an $\mathrm{HDV}$-field with $\widehat{K}$ quasifinite, and let $R / K$ be an algebraic extension. Then $\operatorname{Br}(R)_{p} \neq\{0\}$, for a given $p \in \mathbb{P}$, if and only if $v(R) \neq p v(R)$ and $\widehat{R}(p) \neq \widehat{R}$.

Proof. Suppose first that $\widehat{R}(p) \neq \widehat{R}$ and $v(R) \neq p v(R)$. Then, by Lemma 3.1, there exists a degree $p$ extension $R_{1}$ of $R$ in $R_{\mathrm{ur}}$; also, by assumption, there is $\pi \in K^{*}$ of value $v(\pi) \notin p v(R)$. Fix a generator $\sigma$ of $\mathcal{G}\left(R_{1} / R\right)$ and consider the cyclic $R$-algebra $A=\left(R_{1} / R, \sigma, \pi\right)$ (for the definition of $A$, see, e.g., [17, Sect. 2.5). It is known that $A$ is a nicely semiramified division $R$-algebra of dimension $p^{2}$, in the sense of Jacob-Wadsworth (see [30], page 452 , and further references there). In particular, $R$ equals the centre of $A$ and the Brauer equivalence class of $A$ is an element of order $p$ in $\operatorname{Br}(E)$, which shows that $\operatorname{Br}(R)_{p} \neq\{0\}$.

In the rest of the proof of Lemma 3.5, we assume that $\operatorname{Br}(R)_{p} \neq\{0\}$. Our goal is to show that $\widehat{R}(p) \neq \widehat{R}$ and $v(R) \neq p v(R)$. We first prove that $\widehat{R}(p) \neq \widehat{R}$. As $\widehat{K}$ is quasifinite, $\mathcal{G}_{\widehat{K}}$ is isomorphic to the profinite group $\prod_{\ell \in \mathbb{P}} \mathbb{Z}_{\ell}$, so it follows from Lemma 3.1 and the equality $R_{\mathrm{ur}}=R K_{\mathrm{ur}}$, that if 
$\widehat{R}(p)=\widehat{R}$, then $R \cap K_{\text {ur }}$ contains as a subfield a $\mathbb{Z}_{p}$-extension $\Gamma_{p}$ of $K$. Hence, by Galois theory, condition (c) of Lemma 3.4 holds with respect to $\Gamma_{p} / K$. This requires $\operatorname{Br}\left(\Gamma_{p}\right)_{p}=\{0\}$ and $\operatorname{Br}(R)_{p}=\{0\}$, which is a contradiction proving that $\widehat{R}(p) \neq \widehat{R}$.

We turn to the proof of the assertion that $v(R) \neq p v(R)$. Denote by $R_{0}$ the maximal separable extension of $K$ in $R$. Since $\operatorname{Br}(R)_{p} \neq\{0\}$, Lemma 3.4 yields $\operatorname{Br}\left(R_{0}\right)_{p} \neq\{0\}$ and implies the existence of a finite extension $L$ of $K$ in $R_{0}$, such that $p \nmid\left[L_{1}: L\right]$, for any finite extension $L_{1}$ of $L$ in $R_{0}$. In view of (3.3) (or the isomorphism $v(L) \cong \mathbb{Z}$, see [12], Corollary 14.2.2), this means that $v\left(R_{0}\right) \neq p v\left(R_{0}\right)$, which completes the proof of Lemma 3.5. in the case where $\operatorname{char}(K)=0$. We assume further that $\operatorname{char}(K)=q>0$. Then $R$ is purely inseparable over $R_{0}$, whence finite extensions of $R_{0}$ in $R$ are of $q$-primary degrees. Therefore, (3.3) indicates that if $p \neq q$, then $v(R) \neq p v(R)$, as claimed.

Suppose now that $p=q$, put $R_{v}=R_{v_{R}}$ and $L_{v}=L_{v_{L}}$, fix an algebraic closure $\bar{R}_{v}$ of $R_{v}$, denote by $\bar{R}$ the algebraic closure of $R$ in $\bar{R}_{v}$, identify the field $L_{v}$ with the topological closure of $L$ in $R_{v}$, and put $v^{\prime}=\overline{v_{L}}$. Consider the fields $R^{\prime}=R L_{v}$ and $R_{0}^{\prime}=R_{0} L_{v}$. We first show that $R^{\prime}=R_{0}^{\prime} L^{\prime}$, where $L^{\prime}$ is the maximal purely inseparable extension of $L_{v}$ in $R^{\prime}$. Our proof relies on the fact that, by (3.2) (b), $\left(L_{v}, v^{\prime}\right)$ is a complete discrete valued field with $\widehat{L}_{v}=\widehat{L}$. Observing also that $\widehat{L} / \widehat{K}$ is a finite extension, whence $\widehat{L}$ is a quasifinite field, one obtains from (3.5) (a) (or rather, from the fulfillment of condition (3.4) (b)) that $\left[L_{v}: L_{v}^{p}\right]=p$. As the extension $R_{0}^{\prime} / L_{v}$ is separable (it preserves the separability of $R_{0} / L$ ), this allows to deduce from [5], Lemma 2.12 (and [24], Ch. V, Corollary 6.10) that $\left[R_{0}^{\prime}: R_{0}^{\prime p}\right]=p$. Thus it turns out that, for each $\nu \in \mathbb{N}$, there exists a unique pair $\Lambda_{\nu}, \Lambda_{\nu}^{\prime} \in$ $I\left(\bar{R}_{v} / L_{v}\right)$, such that $\Lambda_{\nu} / L_{v}$ and $\Lambda_{\nu}^{\prime} / R_{0}^{\prime}$ are purely inseparable extensions of degree $p^{\nu}$. Moreover, one obtains the following:

(3.7) (a) $\Lambda_{1}^{p}=L_{v}$, and for each $\nu \in \mathbb{N}, \Lambda_{\nu} \subset \Lambda_{\nu+1}$ and $\Lambda_{\nu+1}^{p}=\Lambda_{\nu}$; also, for any $\pi \in L_{v}^{* p}$ with $v^{\prime}(\pi) \notin p v^{\prime}\left(L_{v}\right)=p v(L), \Lambda_{\nu}=L_{v}\left(\pi_{\nu}\right)$, where $\pi_{\nu} \in \bar{R}_{v}$ is the $p^{\nu}$-th root of $\pi$;

(b) The union $\Lambda_{\infty}$ of the fields $\Lambda_{\nu}, \nu \in \mathbb{N}$, is a perfect field, and $I\left(\Lambda_{\infty} / L_{v}\right)$ equals the set $\left\{\Lambda_{\infty}, L_{v}, \Lambda_{\nu}: \nu \in \mathbb{N}\right\}$ (this description of $I\left(\Lambda_{\infty} / L_{v}\right)$ characterizes the property that $\left[L_{v}: L_{v}^{p}\right]=p$, see Remark 1 in [29], Ch. II, 3.2).

Clearly, $\Lambda_{\infty}$ is the maximal purely inseparable extension of $L_{v}$ in $\bar{R}_{v}$. As $R_{0}^{\prime}$ is separable over $L_{v}$, this implies the set of purely inseparable extensions of $R_{0}^{\prime}$ in $\bar{R}_{v}$ is equal to $I\left(\Lambda_{\infty}^{\prime} / R_{0}^{\prime}\right)$, where $\Lambda_{\infty}^{\prime}=R_{0}^{\prime} \Lambda_{\infty}$ (cf. [24], Ch. V, Corollary 6.10), and for each $\nu \in \mathbb{N}, R_{0}^{\prime} \Lambda_{\nu} / R_{0}^{\prime}$ is a field extension of degree $p^{\nu}$. It is now easy to see that $\Lambda_{\nu}^{\prime}=R_{0}^{\prime} \Lambda_{\nu}, \nu \in \mathbb{N}$, and also, $R_{0}^{\prime}$ and $\Lambda_{\nu}^{\prime}$, $\nu \in \mathbb{N} \cup\{\infty\}$, are all purely inseparable extensions of $R_{0}^{\prime}$ in $\bar{R}_{v}$, so the equality $R^{\prime}=R_{0}^{\prime} L^{\prime}$ is proved.

We continue with the proof of the inequality $v(R) \neq p v(R)$. As noted above, the field extension $R_{0}^{\prime} / L_{v}$ is separable, and since $R^{\prime}=R_{0}^{\prime} L^{\prime}$, it follows that $R^{\prime} / L^{\prime}$ is also separable whereas $R^{\prime} / R_{0}^{\prime}$ is purely inseparable. Identifying the completion of $\left(R_{0}, v_{R_{0}}\right)$ with the topological closure $R_{0, v}$ of $R_{0}$ in $R_{v}$, one obtains similarly that $R_{0, v} \cdot R / R_{0, v}$ is purely inseparable as well. Observe that $\left(L, v_{L}\right),\left(L_{v}, v^{\prime}\right)$ and $R_{0} / L$ satisfy the conditions of Lemma 
3.3. Note also $\left(R_{v}, \overline{v_{R}}\right) /\left(L_{v}, v^{\prime}\right)$ is a valued field extension, so it follows from Lemma 3.3. applied to $\left(L, v_{L}\right),\left(L_{v}, v^{\prime}\right)$ and $R_{0}$, and from the uniqueness of the prolongations of $v_{R_{0}}, v_{R_{0}^{\prime}}^{\prime}$ and $\overline{v_{R_{0}}}$ on $R, R^{\prime}$, and $R_{0, v} R$, respectively (cf. [12], Proposition 14.2.5), that $\left(R^{\prime}, v_{R^{\prime}}^{\prime}\right)$ is an intermediate valued field of $\left(R_{v}, \overline{v_{R}}\right) /\left(R, v_{R}\right)$. This implies the topologies on $R$ and $R^{\prime}$ associated with $v_{R}$ and $v_{R^{\prime}}^{\prime}$, respectively, are induced by the topology of $R_{v}$ (which in turn is determined by $\overline{v_{R}}$, see [12], Theorem 9.3.2). Moreover, $R$ and $R^{\prime}$ are dense in $R_{v}$, and both $\left(R, v_{R}\right)$ and $\left(R^{\prime}, v_{R^{\prime}}^{\prime}\right)$ are Henselian, which guarantees the injectivity of the maps $\pi_{R \rightarrow R_{v}}$ and $\pi_{R^{\prime} \rightarrow R_{v}}$ (cf. [9], Theorem 1). Since, by basic well-known properties of tensor products (cf. 27, Sect. 9.4, Corollary a), $\pi_{R \rightarrow R_{v}}$ equals the composition $\pi_{R^{\prime} \rightarrow R_{v}} \circ \pi_{R \rightarrow R^{\prime}}$, this implies $\pi_{R \rightarrow R^{\prime}}$ is injective, so it follows from the nontriviality of $\operatorname{Br}(R)_{p}$ that $\operatorname{Br}\left(R^{\prime}\right)_{p} \neq\{0\}$. Note further that $\left[L^{\prime}: L_{v}\right]<\infty$. Assuming the opposite, one obtains from (3.7) (b) that $L^{\prime}$ must equal the (perfect) field $\Lambda_{\infty}$. Therefore, $R^{\prime}=R_{0}^{\prime} L^{\prime}$ must also be perfect, which requires $\operatorname{Br}\left(R^{\prime}\right)_{p}=\{0\}$ (cf. [1], Ch. VII, Theorem 22) - a contradiction proving that $\left[L^{\prime}: L_{v}\right]<\infty$.

It is now easy to complete the proof of the inequality $v(R) \neq p v(R)$ (and of Lemma 3.5). Indeed, $\left(L_{v}, v^{\prime}\right)$ is an HDV-field, and we have $\left[L^{\prime}: L_{v}\right]<\infty$, so it follows from [12], Corollary 14.2.2, that $\left(L^{\prime}, v_{L^{\prime}}^{\prime}\right)$ is HDV as well. As $\left[L_{v}: L_{v}^{p}\right]=p$ and $L^{\prime} / L$ is purely inseparable, (3.7) (a) shows that $L^{\prime} / L$ is totally ramified; in particular, $\widehat{L}^{\prime}=\widehat{L}_{v}=\widehat{L}$. Note further that $\widehat{L}$ is a quasifinite field, $R^{\prime} / L^{\prime}$ is a separable extension and $\operatorname{Br}\left(R^{\prime}\right)_{p} \neq\{0\}$. Therefore, $R^{\prime} / L^{\prime}$ violates condition (c) of Lemma 3.4, which enables one to deduce from (3.3) and the inequality $v^{\prime}\left(L^{\prime}\right) \neq p v^{\prime}\left(L^{\prime}\right)$ that $v^{\prime}\left(R^{\prime}\right) \neq p v^{\prime}\left(R^{\prime}\right)$. Since $\left(R^{\prime}, v_{R^{\prime}}^{\prime}\right)$ is an intermediate valued field of $\left(R_{v}, \overline{v_{R}}\right) /\left(R, v_{R}\right)$, whence, $\overline{v_{R}}\left(R_{v}\right)=v^{\prime}\left(R^{\prime}\right)=v(R)$, the inequality $v(R) \neq p v(R)$ now becomes obvious, so Lemma 3.5 is proved.

Lemma 3.5 shows that if $(K, v)$ is an HDV-field with $\widehat{K}$ quasifinite, and $R / K$ is an algebraic extension, then $\operatorname{dim}(R) \leq 1$ if and only if the intersection $S(R) \cap \Sigma(R)$ is the empty set, where $S(R)=\left\{p \in \mathbb{P}: \mathcal{G}(\widehat{R}(p) / \widehat{R}) \cong \mathbb{Z}_{p}\right\}$ and $\Sigma(R)=\{p \in \mathbb{P}: v(R) \neq p v(R)\}$.

Remark 3.6. Let $(K, v)$ be an HDV-field with $\widehat{K}$ quasifinite and $\operatorname{char}(K)=$ $p>0$, and let $R / K$ be any algebraic field extension. Then it follows from (3.3) and Lemma 3.5 that $\operatorname{Br}(R)_{p}=\{0\}$ if and only if one of the conditions (a) and (b) of Lemma 3.4 holds; also, by the proof of Lemma 3.4. the assumption that $\operatorname{Br}(R)_{p}=\{0\}$ ensures that condition (c) is satisfied. The fulfillment of condition (c), however, does not guarantee that $\operatorname{Br}(R)_{p}=\{0\}$ if $K$ has an infinite purely inseparable extension $K^{\prime}$ with $v\left(K^{\prime}\right)=v(K)$ and $\widehat{K}^{\prime}=\widehat{K}$ (see [7], Remark 8.7, for examples of such HDV-fields). Then $\left(K^{\prime}, v_{K^{\prime}}\right)$ is HDV and $\widehat{K}^{\prime}$ is quasifinite, proving that $\operatorname{Br}\left(K^{\prime}\right) \cong \mathbb{Q} / \mathbb{Z}$; hence, $\operatorname{Br}\left(K^{\prime}\right)_{p^{\prime}} \neq\{0\}, p^{\prime} \in \mathbb{P}$. On the other hand, there are fields $R_{n} \in I\left(K^{\prime} / K\right)$, $n \in \mathbb{N}$, such that $\left[R_{n}: K\right]=p^{n}$, for each $n$; therefore, $K^{\prime} / K$ satisfies condition (c) of Lemma 3.4 .

At the end of this Section, note that, for an arbitrary field $R$, the assumption that $\operatorname{Br}(R)_{p}=\{0\}$ is, generally, weaker than conditions (a) and 
(b) of Lemma 3.4. As shown by M. Auslander, the formal power series field $\mathbb{Q}_{\text {sol }}((X))$, where $\mathbb{Q}_{\text {sol }}$ is the compositum of finite Galois extensions of $\mathbb{Q}$ in $\mathbb{Q}_{\text {sep }}$ with solvable Galois groups, satisfies $\operatorname{Br}\left(\mathbb{Q}_{\text {sol }}((X))\right)=\{0\}$ but violates condition (a), for each $p \in \mathbb{P}$ (see [29], Ch. II, 3.1). If, however, $E \in I\left(\mathbb{Q}_{\text {sep }} / \mathbb{Q}\right)$ and $\operatorname{Br}(E)_{p}=\{0\}$, for some $p \in \mathbb{P}$, then $\operatorname{Br}\left(E^{\prime}\right)_{p}=\{0\}$ whenever $E^{\prime} \in I\left(\mathbb{Q}_{\text {sep }} / E\right)$ (see [13], Theorem 4); this can also be deduced from Lemma 3.4 and [29], Ch. II, Proposition 9.

\section{Proof of Theorem 2.2}

Let $(K, v)$ be an HDV-field with $\widehat{K}$ quasifinite, and let $L / K$ be an algebraic extension, $S(L)=\left\{p \in \mathbb{P}: \mathcal{G}(\widehat{L}(p) / \widehat{L}) \cong \mathbb{Z}_{p}\right\}$ and $\Sigma(L)=\{p \in$ $\mathbb{P}: v(L) \neq p v(L)\}$. Then, by Lemma 3.5, $\operatorname{dim}(L) \leq 1$ if and only if $S(L) \cap \Sigma(L)=\emptyset$. Moreover, by Lang's theorem (see [22], Theorem 10), $L$ is a $C_{1}$-field if $S(L)=\emptyset$ and $K_{v}=K$ (the emptiness of $S(L)$ ensures that $K_{\text {ur }} \in I(L / K)$, whence, $L$ preserves the $C_{1}$ type of $\left.K_{\text {ur }}\right)$. Therefore, in this Section, we prove Theorem 2.2 considering fields $L$ with $\operatorname{dim}(L) \leq 1$ and $S(L) \neq \emptyset$. Our proof relies on the following lemma:

Lemma 4.1. Let $(K, v)$ be an $\mathrm{HDV}$-field with $\widehat{K}$ quasifinite, and $S, \Sigma$ be nonempty proper subsets of $\mathbb{P}$. Then there exists an algebraic extension $E / K$, such that $S(E)=S$ and $\Sigma(E)=\Sigma$; hence, $\operatorname{Br}(E)_{p} \neq\{0\}$ if and only if $p \in S \cap \Sigma$.

Proof. Denote by $\widehat{K}(S)$ the compositum of the maximal $p$-extensions $\widehat{K}(p)$, $p \in \mathbb{P} \backslash S$, of $\widehat{K}$ in $\widehat{K}_{\text {sep. }}$. It follows from Lemma 3.1 that $K$ has a Galois extension $K(S)$ in $K_{\text {ur }}$ with $\widehat{K(S)}=\widehat{K}(S)$ and $\mathcal{G}(K(S) / K) \cong \mathcal{G}(\widehat{K}(S) / \widehat{K})$. As $\widehat{K}$ is quasifinite and $K(S)_{\mathrm{ur}}=K_{\mathrm{ur}}$, this enables one to deduce from Galois theory (cf. 24, Ch. VI, Theorem 1.12) and Lemma 3.1 that $\mathcal{G}_{\widehat{K(S)}}$ is isomorphic to the topological group product $\prod_{p \in S} \mathbb{Z}_{p}$. Moreover, $\operatorname{Br}(K(S))_{p}=\{0\}$, $p \in \mathbb{P} \backslash S$, by Lemma 3.5. Now fix an algebraic closure $\bar{K}$ of $K_{\text {sep }}$, take a generator $\pi$ of the ideal $M_{v}(K)$, and for each $t \in \mathbb{P} \backslash \Sigma$, denote by $\Theta_{t}$ the set $\left\{\pi_{t, n}: n \in \mathbb{N}\right\}$, where $\pi_{t, n} \in \bar{K}: n \in \mathbb{N}$, is a sequence defined inductively so that $\pi_{t, 1}=\pi$, and $\pi_{t, n}^{t}=\pi_{t,(n-1)}$, for every $n \geq 2$. It is easily verified that for any $t \in \mathbb{P} \backslash \Sigma$, the extension $K\left(\Theta_{t}\right) / K$ is infinite and finite subextensions of $K$ in $K\left(\Theta_{t}\right)$ are totally ramified of $t$-primary degrees. Therefore, by Lemma 3.5, $\operatorname{Br}\left(K\left(\Theta_{t}\right)\right)_{t}=\{0\}$ and $v\left(K\left(\Theta_{t}\right)\right)=t v\left(K\left(\Theta_{t}\right)\right)$, for $t \in \mathbb{P} \backslash \Sigma$.

Consider now the compositum $E$ of the fields $K(S)$ and $K\left(\Theta_{t}\right), t \in \mathbb{P} \backslash \Sigma$. The described properties of $K(S)$ and $K\left(\Theta_{t}\right), t \in \mathbb{P} \backslash \Sigma$, ensure that $(E, v)$ satisfies the following conditions:

(4.1) (a) Finite extensions of $K(S)$ in $E$ are totally ramified and their degrees are not divisible by any $\lambda \in \Sigma$;

(b) $\mathcal{G}_{\widehat{E}}$ is isomorphic to $\prod_{p \in S} \mathbb{Z}_{p}$, and $v(E) \neq p v(E)$ if and only if $p \in \Sigma$.

Statements (4.1) imply $S(E)=S$ and $\Sigma(E)=\Sigma$, so it follows from Lemma 3.5 that $\operatorname{Br}(E)_{p} \neq\{0\}$ if and only if $p \in S \cap \Sigma$. Lemma 4.1 is proved. 
Next we show that $\mathbb{P}$ possesses subsets $S$ and $\Sigma$ of satisfying the following:

(4.2) (a) $\Sigma$ is infinite, $S \cap \Sigma=\emptyset$, and for each $p \in \Sigma$, there is $k(p) \in \mathbb{Z}$, such that $2 \leq k(p) \leq(p-1) / 2$ and all prime divisors of $k(p) .(p-k(p))$ lie in $S$;

(b) $\operatorname{gcd}\left(k(p) \cdot(p-k(p)), k\left(p^{\prime}\right) \cdot\left(p^{\prime}-k\left(p^{\prime}\right)\right)\right)$ is a 2-primary number, for any $p, p^{\prime} \in \Sigma$ with $p \neq p^{\prime}$.

The proof of (4.2) relies on Dirichlet's theorem about the prime numbers in an arithmetic progression, and on the Chinese Remainder Theorem. Using repeatedly these theorems, one obtains inductively that there exist positive integers $k_{n}, p_{n}, n \in \mathbb{N}$, such that:

(4.3) (a) $k_{1}=2, p_{1}=5$, and for each $n, k_{n} \equiv 2^{n}\left(\bmod 2^{n+1}\right)$ and $p_{n} \in \mathbb{P}$;

(b) If $n \geq 2$, then $k_{n} \equiv 1\left(\bmod \prod_{j=1}^{n-1} p_{j} k_{j} \cdot 2^{-j}\left(p_{j}-k_{j}\right)\right)$,

$p_{n} \equiv 2\left(\bmod \prod_{j=1}^{n-1} p_{j} k_{j} \cdot 2^{-j}\left(p_{j}-k_{j}\right)\right), p_{n} \equiv 1\left(\bmod k_{n}\right)$, and $p_{n} \geq 1+2 k_{n}$.

Let now $\Sigma=\left\{p_{n}: n \in \mathbb{N}\right\}$ and $S=\left\{p \in \mathbb{P}: p \mid k_{n}\left(p_{n}-k_{n}\right)\right.$, for some $\left.n \in \mathbb{N}\right\}$. Arguing by induction on $n$, and using (4.3), one obtains that $p_{n} \notin S$, for any $n$, i.e. $\Sigma \cap S=\emptyset$. Moreover, it is easily verified that $S$ and $\Sigma$ satisfy conditions (4.2), where $k\left(p_{n}\right)=k_{n}$, for each $n \in \mathbb{N}$.

We prove Theorem 2.2. Suppose that $S$ and $\Sigma$ are defined in accordance with (4.3), and $(K, v)$ and $E$ have the properties required by Lemma 4.1 (equivalently, by (4.1) (b)). Fix elements $\pi_{n} \in E^{*}, n \in \mathbb{N}$, of values $v\left(\pi_{n}\right)>$ 0 and $v\left(\pi_{n}\right) \notin p_{n} v(E)$, for any $n$. It follows from (4.1) (b), Galois theory (cf. [24], Ch. VI) and the definition of $S$ that $E$ has cyclic extensions $E_{n}$ and $E_{n}^{\prime}$, such that $\left[E_{n}: E\right]=k_{n},\left[E_{n}^{\prime}: E\right]=p_{n}-k_{n}$ and $E_{n} \cdot E_{n}^{\prime} \subset E_{\text {ur }}$, for each $n \in \mathbb{N}$. Take primitive elements $\xi_{n} \in O_{v}\left(E_{n}\right)$ of $E_{n} / E$ and $\eta_{n} \in O_{v}\left(E_{n}^{\prime}\right)$ of $E_{n}^{\prime} / E$, so that the residue classes $\hat{\xi}_{n} \in \widehat{E}_{n}$ and $\hat{\eta}_{n} \in \widehat{E}_{n}^{\prime}$ are primitive elements of $\widehat{E}_{n}^{\prime} / \widehat{E}$ and $\widehat{E}_{n}^{\prime} / \widehat{E}$, respectively, and consider a system $\bar{X}_{n}=X_{n, i}, i=1, \ldots, k_{n}$, of algebraically independent variables over $E$. In view of Lemma 3.1 and Galois theory, $E_{n}\left(\bar{X}_{n}\right) / E\left(\bar{X}_{n}\right)$ and $E_{n}^{\prime}\left(\bar{X}_{n}\right) / E\left(\bar{X}_{n}\right)$ are cyclic field extensions of degrees $k_{n}$ and $p_{n}-k_{n}$, respectively, and the product $g_{n}\left(\bar{X}_{n}\right)$ of the norms

$$
N_{E_{n}\left(\bar{X}_{n}\right) / E\left(\bar{X}_{n}\right)}\left(\sum_{i=1}^{k_{n}} \xi_{n}^{i-1} X_{n, i}\right) \text { and } N_{E_{n}^{\prime}\left(\bar{X}_{n}\right) / E\left(\bar{X}_{n}\right)}\left(\sum_{i=1}^{k_{n}} \eta_{n}^{i-1} X_{n, i}\right)
$$

is a form of degree $p_{n}$ in the variables $X_{n, i}, i=1, \ldots, k_{n}$, with coefficients in $O_{v}(E)$. Moreover, since $v$ is Henselian, it follows from the inclusion $E_{n} E_{n}^{\prime} \subset E_{\text {ur }}$, the assumptions on $\xi_{n}$ and $\eta_{n}$, and the inequality $k_{n}<p_{n}-k_{n}$ that

(4.4) $g_{n}\left(\bar{\alpha}_{n}\right) \in O_{v}(E)$, for every $k_{n}$-tuple $\bar{\alpha}_{n}=\left(\alpha_{n, 1}, \ldots, \alpha_{n, k_{n}}\right)$ with components $\alpha_{n, i} \in O_{v}(E) ; g_{n}\left(\bar{\alpha}_{n}\right) \in M_{v}(E)$ if and only if $\alpha_{n, i} \in M_{v}(E)$, $i=1, \ldots, k_{n}$.

One also deduces from (4.4) that $v\left(g_{n}\left(\bar{\beta}_{n}\right)\right) \in p_{n} v(E)$, provided that $\bar{\beta}_{n}=\left(\beta_{n, 1}, \ldots, \beta_{n, k_{n}}\right), \beta_{n, i} \in E$, for $i=1, \ldots k_{n}$, and $\beta_{n, i^{\prime}} \neq 0$, for some index $i^{\prime}$. Let now $\widetilde{X}=X_{n, i, j}: i=1, \ldots, k_{n} ; j=1, \ldots, p_{n}$, be a system of $p_{n} k_{n}$ algebraically independent variables over $E$, and let $f_{n}(\tilde{X})=$ $\sum_{j=1}^{p_{n}} g_{n}\left(\widetilde{X}_{n, j}\right) \cdot \pi_{n}^{j}$, where $\tilde{X}_{n, j}=X_{n, i, j}, i=1, \ldots, k_{n}$, for each fixed $j \in$ 
$\left\{1, \ldots, p_{n}\right\}$. Using the noted properties of $g_{n}(\bar{X})$ and the fact that $v\left(\pi_{n}\right) \notin$ $p_{n} v(E)$, one obtains that

(4.5) For any $n \in \mathbb{N}, f_{n}(\tilde{X})$ is an $E$-form of degree $p_{n}$ in $p_{n} k_{n}$ variables, and without a nontrivial zero over $E$; in particular, $E$ is not a $C_{1}$-field.

It follows from (4.1) (b) and Galois theory that if $E^{\prime}$ is a finite extension of $E$, then $S\left(E^{\prime}\right)=S(E)$ and $\Sigma\left(E^{\prime}\right)=\Sigma(E)$. Therefore, $E^{\prime}$ preserves the properties of $E$ described by (4.1) (b), which enables one to show by the method of proving (4.5) that $E^{\prime}$ is not a $C_{1}$-field. Theorem 2.2 is proved.

Theorem 2.2 and our next result exhibit the fact that, for any global or local field $K$, algebraic extensions $E$ of $K$ with $\operatorname{dim}(E) \leq 1$ need not be $C_{1}$-fields.

Corollary 4.2. Let $K$ be a global field, $v$ a Krull valuation of $K$, and $K^{\prime}$ the maximal separable extension of $K$ in $K_{v}$. Then $K^{\prime}$ has an algebraic extension $E^{\prime}$, such that $\operatorname{dim}\left(E^{\prime}\right) \leq 1$ and finite extensions of $E^{\prime}$ are not $C_{1}$-fields.

Proof. Denote by $\bar{v}$ the continuous prolongation of $v$ on $K_{v}$, and by $v^{\prime}$ the valuation of $K^{\prime}$ induced by $\bar{v}$. Observe that $\left(K^{\prime}, v^{\prime}\right)$ is HDV as well as an intermediate valued field of $\left(K_{v}, \bar{v}\right) /(K, v)$ (see (3.2) and the end of Section 1). Hence, $v\left(K^{\prime}\right)=v(K)$ and $\widehat{K}^{\prime}$ equals the residue field of $(K, v)$; in addition, $\widehat{K}^{\prime}$ is finite. Now Corollary 4.2 is proved by applying Theorem 2.2 to $\left(K^{\prime}, v^{\prime}\right)$.

Corollary 4.3. Let $(K, v)$ be an $\mathrm{HDV}$-field with $\widehat{K}$ quasifinite, and let $E$ be an algebraic extension of $K$ with $S(E) \cap \Sigma(E)=\emptyset$. Assume that $k_{1}$ and $k_{2}$ are integers, such that $2 \leq k_{1}<k_{2}$, every divisor $p \in \mathbb{P}$ of $k_{1} k_{2}$ lies in $S(E)$, and every $p^{\prime} \in \mathbb{P}$ dividing $k_{1}+k_{2}$ lies in $\Sigma(E)$. Then $\operatorname{dim}(E) \leq 1$ and there exists an $E$-form $f$ of degree $k_{1}+k_{2}$ in $k_{1}\left(k_{1}+k_{2}\right)$ variables, without a nontrivial zero over $E$; in particular, $E$ is not a $C_{1}$-field and $S(E)$ contains at least 2 elements.

Proof. The inequality $\operatorname{dim}(E) \leq 1$ follows from Lemma3.5 and the condition on $S(E) \cap \Sigma(E)$. Also, our assumptions show that $\operatorname{gcd}\left(k_{1}, k_{2}\right)=1, S(E)$ is of cardinality $\geq 2$, and there exist fields $E_{j} \in I\left(E_{\mathrm{ur}} / E\right)$ with $\left[E_{j}: E\right]=k_{j}$, for $j=1,2$. Using the cyclicity of finitely-generated subgroups of $\mathbb{Q}$, one obtains that the set $\left(k_{1}+k_{2}\right) v(E)=\left\{\left(k_{1}+k_{2}\right) \gamma: \gamma \in v(E)\right\}$ is a subgroup of $v(E)$ of index $k_{1}+k_{2}$, and the group $v(E) /\left(k_{1}+k_{2}\right) v(E)$ is cyclic. This allows to define (like in the proof of (4.5)) an $E$-form as required by Corollary 4.3 .

Note that if $k_{1}$ and $k_{2}$ are integers with $2 \leq k_{1}<k_{2}$ and $\operatorname{gcd}\left(k_{1}, k_{2}\right)=1$, then $\operatorname{gcd}\left(k_{1} k_{2}, k_{1}+k_{2}\right)=1$. This implies the existence of various subsets $S$ and $\Sigma$ of $\mathbb{P}$, such that $S \cap \Sigma=\emptyset$, and $p \in S, p^{\prime} \in \Sigma$, for any pair $p, p^{\prime} \in \mathbb{P}$ satisfying $p \mid k_{1} k_{2}$ and $p^{\prime} \mid k_{1}+k_{2}$. Therefore, Lemma 4.1, Corollary 4.3 and the proof of Theorem 2.1 make it easy to demonstrate the fact that fields 
of dimension $\leq 1$ and without the property $C_{1}$ are not uncommon for the class of algebraic extensions of any local or global field.

Remark 4.4. It is known that PAC fields have dimension $\leq 1$ (see [15], Theorem 11.6.4, Corollary 11.2.5). Note also that $\operatorname{cd}\left(\mathcal{G}_{F}\right) \leq 1$, for any field $F$ with $\operatorname{dim}(F) \leq 1$. Conversely, if $G$ is a profinite group with $\operatorname{cd}(G) \leq$ 1 , then $G \cong \mathcal{G}_{F^{\prime}}$, for some perfect PAC field $F^{\prime}$ (see [25], page 44; [15], Corollary 23.1.2). The question of whether almost perfect PAC fields are of type $C_{1}$ seems to be open. As global fields are almost perfect, it relates Problem 2.3 to the main topic of this paper. A conjecture of Ax predicts, for perfect PAC fields, that the answer to the question is affirmative. It has been proved in characteristic zero (in [21]), and for a perfect PAC field $\Phi_{\ell}$ with $\mathcal{G}_{\Phi_{\ell}}$ a pro- $\ell$-group, where $\ell \in \mathbb{P}$ (see [32, Sect. 3 ). These results imply one cannot prove that a field $F$ with $\operatorname{dim}(F) \leq 1$ and $\operatorname{char}(F)=0$ is not of type $C_{1}$, using only topological invariants of $\mathcal{G}_{F}$.

Corollary 4.5. With assumptions being as in the proof of Theorem 2.2, for each finite extension $E^{\prime} / E$ of odd degree, there is a finite subset $m\left(E^{\prime}\right) \subset \mathbb{N}$, such that the forms $f_{n}, n \notin m\left(E^{\prime}\right)$, are without nontrivial zeroes over $E^{\prime}$.

Proof. We first show that the norm group $N(\Lambda / E)$ equals $N\left(\Lambda E^{\prime} / E^{\prime}\right) \cap E^{*}$, for any $\Lambda \in \mathrm{Fe}(E)$ and each finite extension $E^{\prime}$ of $E$ in an algebraic closure of $E_{\text {sep }}$, such that $\operatorname{gcd}\left(t, t^{\prime}\right)=1$, where $t=[\Lambda: E]$ and $t^{\prime}=\left[E^{\prime}: E\right]$. It is easily verified that $\Lambda \cap E^{\prime}=E,\left[\Lambda E^{\prime}: E^{\prime}\right]=[\Lambda: E]$ and the restriction of the mapping $N_{\Lambda E^{\prime} / E^{\prime}}$ on $\Lambda$ coincides with $N_{\Lambda / E}$; therefore, $N(\Lambda / E) \subseteq$ $N\left(\Lambda E^{\prime} / E^{\prime}\right) \cap E^{*}$. Note also that $N_{\Lambda E^{\prime} / E}$ equals the compositions $N_{\Lambda / E} \circ$ $N_{\Lambda E^{\prime} / \Lambda}$ and $N_{E^{\prime} / E} \circ N_{\Lambda E^{\prime} / E^{\prime}}$, which yields $N\left(\Lambda E^{\prime} / E\right) \subseteq N(\Lambda / E)$ and $\alpha^{t^{\prime}} \in$ $N\left(\Lambda E^{\prime} / E\right)$, for all $\alpha \in N\left(\Lambda E^{\prime} / E^{\prime}\right) \cap E^{*}$. As $E^{* t} \subseteq N(\Lambda / E)$, these facts show that $N\left(\Lambda E^{\prime} / E^{\prime}\right) \cap E^{*} \subseteq N(\Lambda / E)$, so it turns out that $N(\Lambda / E)=$ $N\left(\Lambda E^{\prime} / E^{\prime}\right) \cap E^{*}$, as claimed. It follows from this result that, for any $n \in \mathbb{N}$, the form $f_{n}$ defined over $K$ as in the proof of Theorem 2.2, can be defined essentially in the same way over any finite extension $K_{n}$ of $K$ of degree relatively prime to $p_{n} k_{n}\left(p_{n}-k_{n}\right)$; hence, $f_{n}$ does not possess a nontrivial zero over $K_{n}$. Since, by $(4.3), \operatorname{gcd}\left(p_{m} k_{m}\left(p_{m}-k_{m}\right), p_{n} k_{n}\left(p_{n}-k_{n}\right)\right)$ is equal to $2^{m}$ if $m, n \in \mathbb{N}$ and $m<n$, this proves Corollary 4.5.

Our next result allows us to lift the restriction on $\left[E^{\prime}: E\right]$ in Corollary 4.5 :

Proposition 4.6. In the setting of Theorem 2.2, the algebraic extension $E$ of $K$ can be chosen so that $\operatorname{dim}(E) \leq 1$ and there exist $E$-forms $f_{n}, n \in \mathbb{N}$, without nontrivial zeroes over $E$, which satisfy the following conditions:

(a) $\operatorname{deg}\left(f_{n}\right)=p_{n}$ and $f_{n}$ depends on $p_{n} t_{n}$ variables, for each $n \in \mathbb{N}$ and some $p_{n}, t_{n} \in \mathbb{P}$, such that $t_{n}<p_{n} / 3$;

(b) The sequence $p_{n} t_{n}, n \in \mathbb{N}$, increases and consists of odd pairwise relatively prime numbers;

(c) For any finite extension $E^{\prime} / E$, there is a finite subset $m\left(E^{\prime}\right)$ of $\mathbb{N}$, such that none of the forms $f_{n}, n \notin m\left(E^{\prime}\right)$, has a nontrivial zero over $E^{\prime}$. 
To prove Proposition 4.6 we need the following lemma:

Lemma 4.7. For each finite subset $P$ of $\mathbb{P}$, there exists $n(P) \in \mathbb{N}$, such that every odd integer $N>n(P)$ is presentable as a sum of three distinct prime numbers greater than any element of $P$.

Proof. This follows from the fact that for any $\alpha \in \mathbb{R}$ with $0<\alpha<1$, there exists $M(\alpha) \in \mathbb{R}$, such that each integer $N>M(\alpha)$ equals the sum $N=p_{1}+p_{2}+p_{3}$, for some $p_{i} \in \mathbb{P}, i=1,2,3$, depending on $N$ so that $N^{\alpha}<$ $p_{1}<p_{2}<p_{3}$. The fact itself has been established by Ax and deduced from Vinogradov's theorem on the Ternary Goldbach Problem (see [4, Lemma 2]).

Proof of Proposition 4.6. Proceeding by induction on $n$, and using Lemma 4.7, one proves the existence of 4 -tuples $\left(t_{n}, \theta_{n}, y_{n}, p_{n}\right), n \in \mathbb{N}$, satisfying the following conditions, for each index $n$ :

(4.6) (a) $t_{n}, \theta_{n}, y_{n}$ and $p_{n}$ lie in $\mathbb{P}$, and $t_{n}+\theta_{n}+y_{n}=p_{n}$;

(b) $2<t_{n}<\theta_{n}<y_{n}$ and $p_{n}<t_{n+1}$.

The rest of our proof goes along the lines drawn in the concluding part of the proof of Theorem 2.2 (after (4.3)), so we present only its main steps and omit details. Put $S=\left\{t_{n}, \theta_{n}, y_{n}: n \in \mathbb{N}\right\}$ and $\Sigma=\left\{p_{n}: n \in \mathbb{N}\right\}$. It follows from (4.6) that $S \cap \Sigma=\emptyset$; also, by Lemma 4.1, there is an algebraic extension $E$ of $K$ with $S(E)=S$ and $\Sigma(E)=\Sigma$. Therefore, $\operatorname{dim}(E) \leq 1$ and conditions (4.1) (b) hold, which ensures the existence of cyclic extensions $T_{n}, \Theta_{n}$ and $Y_{n}, n \in \mathbb{N}$, of $E$ in $E_{\text {ur }}$ of degrees $\left[T_{n}: E\right]=t_{n},\left[\Theta_{n}: E\right]=$ $\theta_{n}$ and $\left[Y_{n}: E\right]=y_{n}$, for each $n$. Fix primitive elements $\xi_{n} \in O_{v}\left(T_{n}\right)^{*}$, $\eta_{n} \in O_{v}\left(\Theta_{n}\right)^{*}$, and $\delta_{n} \in O_{v}\left(Y_{n}\right)^{*}$ of $T_{n} / E, \Theta_{n} / E$ and $Y_{n} / E$, respectively, so that the residue classes $\hat{\xi}_{n} \in \widehat{T}_{n}, \hat{\eta}_{n} \in \widehat{\Theta}_{n}$ and $\hat{\delta}_{n} \in \widehat{Y}_{n}$ be primitive elements of $\widehat{T}_{n} / \widehat{E}, \widehat{\Theta}_{n} / \widehat{E}$ and $\widehat{Y}_{n} / \widehat{E}$, respectively. Take a system $\bar{X}_{n}=X_{n, i}$, $i=1, \ldots, t_{n}$, of algebraically independent variables over $E$, and let $g_{n}\left(\bar{X}_{n}\right)$ be the product of the norms

$$
\begin{gathered}
N_{T_{n}\left(\bar{X}_{n}\right) / E\left(\bar{X}_{n}\right)}\left(\sum_{i=1}^{t_{n}} \xi_{n}^{i-1} X_{n, i}\right), N_{\Theta_{n}\left(\bar{X}_{n}\right) / E\left(\bar{X}_{n}\right)}\left(\sum_{i=1}^{t_{n}} \eta_{n}^{i-1} X_{n, i}\right) \\
\text { and } N_{Y_{n}\left(\bar{X}_{n}\right) / E\left(\bar{X}_{n}\right)}\left(\sum_{i=1}^{t_{n}} \delta_{n}^{i-1} X_{n, i}\right) .
\end{gathered}
$$

Clearly, $g_{n}\left(\bar{X}_{n}\right) \in O_{v}(E)\left[\bar{X}_{n}\right]$ and the specializations $g_{n}\left(\bar{\alpha}_{n}\right)$ of $g_{n}\left(\bar{X}_{n}\right)$, where $\bar{\alpha}_{n}=\left(\alpha_{n, 1}, \ldots, \alpha_{n, t_{n}}\right)$ and all $\alpha_{n, i} \in O_{v}(E)$, are subject to the restrictions of (4.4) (here $k_{n}$ is replaced by $t_{n}$ ). Also, $g_{n}\left(\bar{X}_{n}\right)$ is an $E$ form of degree $p_{n}$, such that $v\left(g_{n}\left(\bar{\beta}_{n}\right)\right) \in p_{n} v(E)$ if $\bar{\beta}_{n}=\left(\beta_{n, 1}, \ldots, \beta_{n, t_{n}}\right)$, $\beta_{n, i} \in E, i=1, \ldots, t_{n}$, and $\beta_{n, i^{\prime}} \neq 0$, for some $i^{\prime}$. Now fix $n$, observe that $v(E) \neq p_{n} v(E)$, take some $\pi_{n} \in E^{*}$ of value $v\left(\pi_{n}\right)>0$ and $v\left(\pi_{n}\right) \notin p_{n} v(E)$, and consider a system $\widetilde{X}=X_{n, i, j}: i=1, \ldots, t_{n} ; j=1, \ldots, p_{n}$, of $p_{n} t_{n}$ algebraically independent variables over $E$. It follows from the preceding observations on $g_{n}\left(\bar{X}_{n}\right)$ that the polynomial $f_{n}(\widetilde{X})=\sum_{j=1}^{p_{n}} g_{n}\left(\widetilde{X}_{n, j}\right) \cdot \pi_{n}^{j}$ has the properties described by (4.5) (with $t_{n}$ instead of $k_{n}$ ), where $\widetilde{X}_{n, j}=X_{n, i, j}$, 
$i=1, \ldots, t_{n}$, for each fixed $j \in\left\{1, \ldots, p_{n}\right\}$. Moreover, arguing as in the proof of Corollary 4.5, one obtains that $f_{n}$ is without nontrivial zeroes over any extension of $E$ of degree relatively prime to the product $p_{n} t_{n} \theta_{n} y_{n}$. Since (4.6) implies $t_{n}<p_{n} / 3$ and $\operatorname{gcd}\left(p_{m} t_{m} \theta_{m} y_{m}, p_{n} t_{n} \theta_{n} y_{n}\right)=1$, for $m \in \mathbb{N}$, $m \neq n$, this completes our proof.

Remark 4.8. The problem of finding relations between Diophantine properties of a field $E$ and the sequence $\operatorname{cd}_{p}\left(\mathcal{G}_{E}\right), p \in \mathbb{P}$, has attracted lasting interest in the study of some modifications of the $C_{i}$-condition, introduced in [19]. The research on this topic focuses on several conjectures stated in [19]. One of them claims that a perfect field $E$ is of type $C_{1}$, provided $\operatorname{dim}(E) \leq 1$ and $\mathcal{G}_{E}$ is a pro-p-group, for some $p \in \mathbb{P}$ (and so suggests a generalization of the Ax conjecture noted in Remark 4.4). The stated generalization need not be true, see [11, 10], but it seems to be unknown whether it holds in case $E$ is an algebraic extension of $\mathbb{Q}$ or $\mathbb{Q}_{\ell}$, for a fixed $\ell \in \mathbb{P}$. We refer the reader to [32] and [18, for more results on other modifications of the $C_{i}$-condition, considered in [19].

Note finally that the present paper leaves open the question of whether a Galois extension $E$ of a global or local field $K$ with $\operatorname{dim}(E) \leq 1$ is a $C_{1^{-}}$field. As shown in [8], if $K$ is a local field, $\operatorname{char}(\widehat{K})=q$ and $\operatorname{dim}(E) \leq 1$, then $S(E) \subseteq\{q\}$, i.e. $\widehat{E}(p)=\widehat{E}$, for all $p \in \mathbb{P} \backslash\{q\}$. This, combined with Lemma 3.5, implies that if $v(E) \neq q v(E)$, then $I(E / K)$ contains a $K$-isomorphic copy of $K_{\text {ur }}$. Since, by Lang's theorem (referred to at the beginning of this Section), $K_{\text {ur }}$ is of type $C_{1}$, it follows that so is $E$. On the other hand, our research and the stated result of [8] indicate that the method of proving Theorem 2.2 does not lead to Galois extensions $L$ of $K$ satisfying the conditions $v(L)=q v(L)$ and $\operatorname{dim}(L) \leq 1$, which are not $C_{1}$-fields.

Acknowledgements. The author wishes to thank the referee for a number of helpful suggestions, used for improving the presentation of this research as a whole, and particularly, of the proofs of Lemmas 3.4, 3.5 and Corollary 4.5, The research itself has partially been supported by the Bulgarian National Science Fund under Grant KP-06 N 32/1 of 07.12.2019.

\section{REFERENCES}

[1] A.A. Albert, Structure of Algebras. Amer. Math. Soc. Coll. Publ., 24, Amer. Math. Soc., XII, New York, 1939.

[2] G.I. Arkhipov, A.A. Karatsuba, Local representation of zero by a form. Izv. Akad. Nauk SSSR Ser. Mat. 45 (1981), No. 5, 948-961, 1198.

[3] J. Ax, A field of cohomological dimension 1 which is not $C_{1}$. Bull. Amer. Math. Soc. 71 (1965), 717.

[4] J. Ax, Proof of some conjectures on cohomological dimension. Proc. Amer. Math. Soc. 16 (1965), 1214-1221.

[5] N. Bhaskhar, B. Haase, Brauer p-dimension of complete discretely valued fields, Trans. Amer. Math. Soc. 373 (2020), 3709-3732.

[6] Z.I. Borevich, I.R. Shafarevich, Number Theory. Third complemented edition. "Nauka", Moscow, 1985 (Russian).

[7] I.D. Chipchakov, On the residue fields of Henselian valued stable fields. J. Algebra 319 (2008), 1, 16-49. 
[8] I.D. Chipchakov, On fields of dimension one that are Galois extensions of a global or local field. Preprint, arXiv:2011.11135 [math.NT].

[9] P.M. Cohn, On extending valuations in division algebras. Stud. Sci. Math. Hungar. 16 (1981), 65-70.

[10] J.-L. Colliot-Thélène, Fields of cohomological dimension one versus $C_{1}$-fields. Algebra and number theory, 1-6, Hindustan Book Agency, Delhi, 2005.

[11] J.-L. Colliot-Thélène, D.A. Madore, Surfaces de del Pezzo sans point rationnel sur un corps de dimension cohomologique un. J. Inst. Math. Jussieu 3 (2004), No. 1, 1-16.

[12] I. Efrat, Valuations, Orderings, and Milnor K-Theory. Math. Surveys and Monographs 124, Amer. Math. Soc., Providence, RI, 2006.

[13] B. Fein, M. Schacher, Brauer groups of fields algebraic over $\mathbb{Q}$. J. Algebra 43 (1976), 1, 328-337.

[14] G. Frey, Pseudo algebraically closed fields with non-archimedean real valuations. J. Algebra 26 (1973), 202-207.

[15] M. Fried, M. Jarden, Field Arithmetic. Third edition. Revised by Jarden. Ergebnisse der Math. und ihrer Grenzgebiete, 3. Folge, 11, Springer, Berlin, 2008.

[16] W.-D. Geyer, M. Jarden, Non-PAC fields whose Henselian closures are separably closed. Math. Res. Lett. 8 (2001), No. 4, 509-519.

[17] Ph. Gille, T. Szamuely, Central Simple Algebras and Galois Cohomology. Cambridge Studies in Advanced Mathematics, 101. Cambridge Univ. Press, Cambridge, 2006.

[18] D. Izquierdo, On a conjecture of Kato and Kuzumaki. Algebra Number Theory 12 (2018), No. 2, 429-454.

[19] K. Kato, T. Kuzumaki, The dimension of fields and algebraic K-theory. J. Number Theory 24 (1986), No. 2, 229-244.

[20] J. Koenigsmann, Products of absolute Galois groups. Int. Res. Math. Not. 2005 (2005), No. 24, 1465-1486.

[21] J. Kollár, A conjecture of Ax and degenerations of Fano varieties, Isr. J. Math. 162 (2007), 235-251.

[22] S. Lang, On quasi algebraic closure. Ann. Math. (2) 55 (1952), 373-390.

[23] S. Lang, Algebraic Numbers. Addison-Wesley Series in Mathematics. Reading, Mass. etc.: Addison-Wesley Publishing Company, Inc. IX, 1964.

[24] S. Lang, Algebra. Revised 3rd ed., Graduate Texts in Math., vol. 211, Springer, New York, 2002.

[25] A. Lubotzky, L. van den Dries, Subgroups of free profinite groups and large subfields of $\tilde{\mathbb{Q}}$. Isr. J. Math. 39 (1981), 25-45.

[26] J. Neukirch, Über eine algebraische Kennzeichnung der Henselkörper. J. reine angew. Math. 231 (1968), 75-81.

[27] R. Pierce, Associative Algebras. Graduate Texts in Math., vol. 88, Springer-Verlag, New York-Heidelberg-Berlin, 1982.

[28] J.-P. Serre, Local Fields. Transl. from the French by M.J. Greenberg, Graduate Texts in Math., 67, Springer-Verlag, VIII, New York-Berlin, 1979.

[29] J.-P. Serre, Galois Cohomology, Transl. from the French by Patrick Ion, SpringerVerlag, X, Berlin-Heidelberg-New York, 1997.

[30] J.-P. Tignol, A.R. Wadsworth, Value Functions on Simple Algebras, and Associated Graded Rings. Springer Monographs in Math., Springer, Cham-Heidelberg-New YorkDordrecht-London, 2015.

[31] A. Weil, Basic Number Theory. Reprint of the 2nd ed. 1973. Classics in Mathematics. Springer-Verlag, Berlin, 1995.

[32] O. Wittenberg, Sur une conjecture de Kato et Kuzumaki concernant les hypersurfaces de Fano. Duke Math. J. 164 (2015), No. 11, 2185-2211.

Institute of Mathematics and Informatics, Bulgarian Academy of Sciences, 1113 Sofia, Bulgaria: E-MAIl AdDrEss: CHIPCHAK@MATH.BAS.BG 